\title{
DNA repair and synthetic lethality
}

\author{
Gong-she Guo ${ }^{1}$, Feng-mei Zhang ${ }^{1}$, Rui-jie Gao ${ }^{2}$, Robert Delsite ${ }^{4}$, Zhi-hui Feng ${ }^{1,3 *}$, Simon N. Powell ${ }^{4 *}$ \\ ${ }^{1}$ School of Public Health, Shandong University, Jinan 250012, China; ${ }^{2}$ Second People's Hospital of Weifang, Weifang \\ 261041, China; ${ }^{3}$ Department of Radiation Oncology, Washington University in St Louis, St Louis MO 63108, USA; \\ ${ }^{4}$ Department of Radiation Oncology, Memorial Sloan Kettering Cancer Center, New York NY 10065, USA
}

Tumors often have DNA repair defects, suggesting additional inhibition of other DNA repair pathways in tumors may lead to synthetic lethality. Accumulating data demonstrate that DNA repair-defective tumors, in particular homologous recombination (HR), are highly sensitive to DNA-damaging agents. Thus, HR-defective tumors exhibit potential vulnerability to the synthetic lethality approach, which may lead to new therapeutic strategies. It is well known that poly (adenosine diphosphate (ADP)-ribose) polymerase (PARP) inhibitors show the synthetically lethal effect in tumors defective in BRCA1 or BRCA2 genes encoded proteins that are required for efficient HR. In this review, we summarize the strategies of targeting DNA repair pathways and other DNA metabolic functions to cause synthetic lethality in HR-defective tumor cells.

Keywords: DNA repair; homologous recombination; synthetic lethality; BRCA; Rad52

International Journal of Oral Science (2011) 3: 176-179. doi: 10.4248/IJOS11064

\section{Introduction}

Synthetic lethality is defined as a genetic combination of mutations in two or more genes that leads to cell death, whereas a mutation in any one of the genes does not [1]. This phenomenon was first discovered from the investigation of fruit flies in 1922. Since the identical consequence was also found in another species, Drosphila pseudoobscura, the concept of "synthetic lethality" was established. In addition, it would be called synthetic sick when a genetic combination of mutations doesn't cause deadly damage; however, these combinations are often categorized together with synthetic lethal interactions [1-2].

The concept of synthetic lethality in the DNA damage response has recently grown in popularity with the finding that poly (adenosine diphosphate (ADP)-ribose)

\footnotetext{
*Correspondence: Zhi-hui Feng and Simon N. Powell

Tel: 0013147475451 (Feng); 0012126393639 (Powell)

Fax: 0013143629790 (Feng); 0016468883200 (Powell)

E-mail: fengzhihui@sdu.edu.cn (Feng); powells@mskcc.org (Powell)

Received 18 June 2011; Accepted 10 July 2011
}

polymerase (PARP) inhibitors are specifically toxic to homologous recombination (HR)-defective cells [3-4]. Playing a key role in maintaining genetic stability, HR is a major repair pathway for double-strand breaks (DSBs) utilizing undamaged homologous DNA sequence [5-6]. In this review, we summarize the strategies of targeting DNA repair pathways and other DNA metabolic functions to cause synthetic lethality in HR-defective tumor cells, with the hope that they result in tumor-cell specific cancer therapy.

Targeting a specific DNA repair pathway for synthetic lethality

There are multiple pathways of DNA repair, which can partially compensate for each other [5]. Apart from $\mathrm{HR}$, non-homologous end joining (NHEJ) is the major alternative DSB repair mechanism. NHEJ modifies the broken DNA ends prior to ligation, resulting in a mutagenic change at the break site [6]. Nucleotide and base excision repair plus DNA mismatch repair are also important repair pathways in response to DNA damage affecting a single strand of DNA, where the undamaged 
strand is used as the template for repair [7]. Cancer cells often have DNA repair defects, suggesting additional inhibition of other DNA repair pathway in cancer cells may achieve synthetic lethality. For HR-defective tumors, which may be more prevalent than was first thought, there are a number of new avenues for developing cancer therapy, which exploit the concept of synthetic lethality, and which are currently being tested in clinical trials [6].

Inhibition of PARP, a key element for base excision repair pathway, can have synthetic lethality in tumors with defective breast cancer susceptibility genes (BRCA1, BRCA2), which are required for efficient HR repair [3-4]. The PARP protein has the ability to bind singlestrand breaks (SSBs) and facilitate SSBs repair [8]. Inhibition of PARP protein can result in persistent SSBs, perhaps with the PARP protein attached to the SSB and preventing alternative pathways of SSB repair [9]. Once the replication fork encounters an SSB, the lesion can be converted into a DSB requiring for HR for repair. This type of DSB will not be repaired in HR-defective cells. NHEJ may not be an effective alternative pathway for this special type of one-ended DSB that occurs during DNA replication. If PARP inhibitors are present, the number of open SSB is increased, resulting in a greater likelihood of replication-associated DSB, which in turn will produce chromatid breaks and exchanges, resulting in cell death. Several PARP inhibitors have now been used in clinical trials for the treatment of breast and ovarian cancer [6].

Cells with defective HR are vulnerable to synthetic lethality if other DNA repair mechanisms in the HRdefective cells are inhibited. The DNA repair protein Rad52 plays a key role in the HR of Saccharomyces cerevisiae [10]. However, knockout of the Rad52 gene in vertebrates results in mild phenotypes, without obvious defects in HR, suggesting that Rad52 may be redundant for HR in higher eukaryotes [11-12]. Our recent publication has indicated there are two, probably independent, pathways of Rad51-dependent HR in mammalian cells [13]: the Rad52 pathway, which is the only pathway of HR in yeast cells, and the BRCA2 pathway, which is found extensively in the animal and plant kingdoms. Many species have both pathways of repair, but some have only Rad52 (such as yeast) and some have only BRCA2 (such as Drosophila melanogaster and Caenorhabditis elegans). The role of Rad52 in the repair of DSB by HR and in overcoming DNA-replication fork stalling is masked when BRCA2 is present. Rad52 mediates Rad51dependent repair and is an alternative HR pathway that is independent of BRCA2. There are potentially lethal effects of inactivating Rad52 in BRCA2-deficient cells that could provide another new specific treatment resulting in the synthetic lethality of a subset of breast cancers.

Germline mutations in BRCA1 or BRCA2 gene result in a cumulative lifetime risk of developing hereditary breast and ovarian cancer. However, the mutations of BRCA gene in sporadic breast cancers are not frequently examined, and it has been determined that both BRCA1 mRNA and protein expression are significantly downregulated in sporadic breast cancer and ovarian cancer cases [14]. Increasing evidence suggests that the BRCA and Fanconi anemia (FA) pathways may be inactivated by multiple mechanisms in a substantial proportion of sporadic breast cancers, and that these cancers could be effectively BRCA-deficient, which has been called "BRCAness" [15]. These non-mutational mechanisms of BRCAdeficiency in sporadic breast cancer and ovarian cancer are still unclear. Our unpublished data indicate that BRCA1 and BRCA2 work in the same HR repair pathway, and that BRCA2 protein expression is regulated by functional BRCA1. These observations suggest that nonfunctional BRCA1 will lead to BRCA2 protein instability, resulting in additional BRCA2-mediated HR repair pathway defects. These data raise the question of whether the inhibition of Rad52-mediated HR pathway would be synthetically lethal in tumors with down-regulated BRCA1 or BRCA2 protein expression. This hypothesis would expand the potential number of tumors that can be targeted using a synthetic lethality strategy for BRCAdeficient tumors and Rad52. The working model of BRCA-Rad52 and synthetic lethality is shown in Figure 1.

\section{Targeting genes regulating cell cycle checkpoints for synthetic lethality}

In response to DNA damage, both DNA repair and cell cycle checkpoint pathways will be activated. Activation of checkpoints prevents the progression of cells to the next phase of the cycle allowing time for repair of the damaged DNA. The synthetic lethality strategy for cancer therapy is that inhibiting cell cycle checkpoints will result the accumulation of damaged DNA in cells with pre-existing checkpoint or repair defects. The tumor suppressor p53 is required for controlling the G1/S checkpoint and is frequently inactivated in human cancer cells, especially in BRCA1- or BRCA2-associated tumors (unpublished results) [16]. Thus, targeting the checkpoint proteins required for S- and G2 in HR-defective cells is a potential strategy for killing cancer cells [17]. The ataxia telangiectasia mutated (ATM)-related kinase (ATR)-cell cycle checkpoint kinase $1(\mathrm{Chk} 1)$ pathway is responsible for controlling S- and G2 phase checkpoint in response 
to DNA damage [6]. It has been demonstrated that Chk1 inhibitors are able to sensitize p53 mutant human cells [17-18] and FA-mediated repair pathway deficient tumors [19], as several Chk1 inhibitors were used for a phase I / II clinical trial. Apart from the inhibition of Chk1, the inhibition of another G2 checkpoint kinase, WEE1, displayed higher cytotoxicity to p53-deficient cells [20]. Inhibition of the ATM kinase, which also functions in cell cycle checkpoints, leads to the radiosensitization of cancer cells [21].

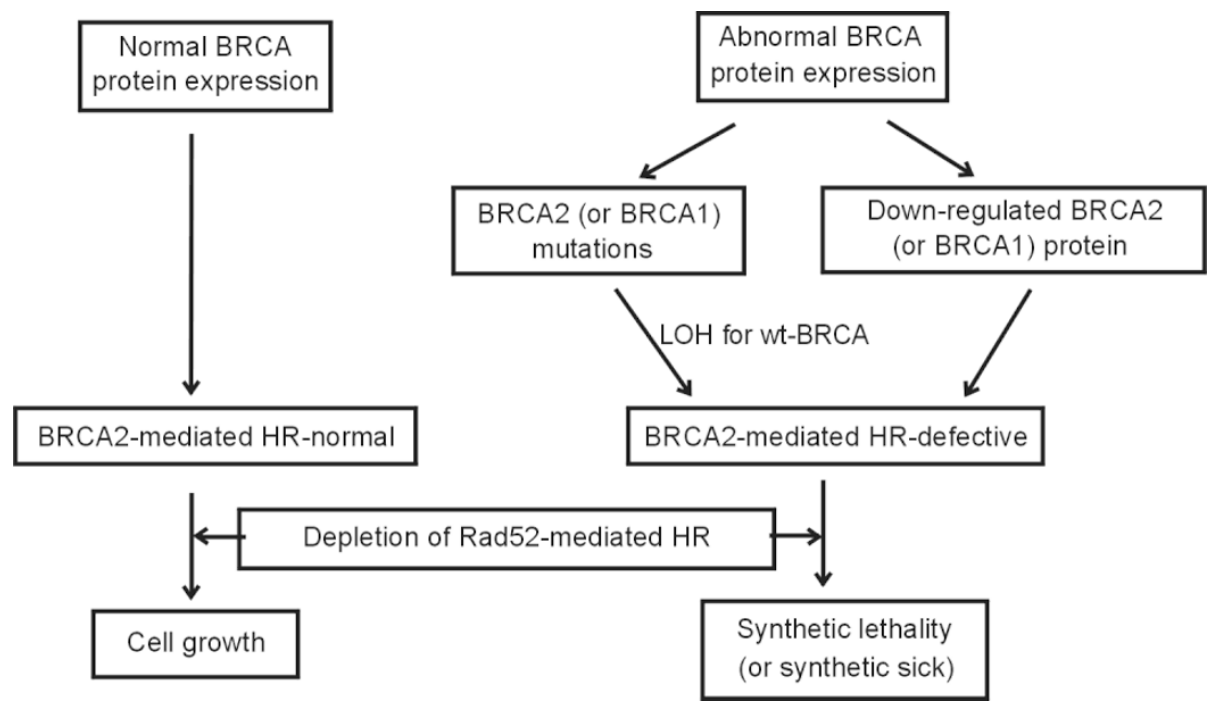

Figure 1 Schematic representation of the synthetic lethality of BRCA-Rad52

The ATR-induced S phase cell cycle checkpoint suppresses both the formation of recombination foci and the recombinational repair of DSBs at chromosome breaks [22]. Chk1 plays a key role in signaling to protect cells against lethal DNA lesions, perhaps in part by regulating HR [23]. Many cancer cells have lost the protection against DNA damage afforded by the $\mathrm{p} 53$ protein, so the repair-defective cancer cells will often have minimal protection against DNA damage persisting well into the S-phase of the cell cycle [24]. The ATR-Chk1 pathway is therefore the only protection remaining in the cancer cells against the effects of DNA damage: targeting this pathway has the potential for significant therapeutic gain in the checkpoint and repair defective cancer cells. However, the precise relationship between cell cycle checkpoint proteins and the BRCA-pathway, and the mechanism of how it regulates HR, is poorly understood. Targeting cell cycle checkpoint proteins in DNA repairdefective tumors is an additional strategy of synthetic lethality in cancer therapy.

\section{Targeting DNA replication}

Double-stranded DNA lesions produced at replication forks are substrates for HR repair [5]. DNA-damaging agents that interfere with DNA replication are employed for the treatment of HR-defective tumors. DNA replication processes that are targeted by chemotherapeutic drugs include antimetabolites, topoisomerase poisons, DNA cross-linking agents, and hydroxyurea (HU) [6, 25]. The initial rationale for their use was the selective targeting of rapidly dividing cancer cells. The DNA lesions induced by these DNA-damaging agents cannot be efficiently repaired in HR-defective cells. For example, HU can deplete the cells of dNTPs, which results in stalled replication forks, which then collapse to form DNA DSB. Current data [25] indicates that the phosphorylated replication protein A2 (RPA2)-p plays an important role in linking replication arrest to $\mathrm{HR}$, and RPA2-p-defective cells are hypersensitive to HU treatment. Targeting DNA replication in DNA repair-defective cells could also be a viable synthetic lethality strategy in cancer cells.

In summary, synthetic lethality approaches to therapeutic interventions in DNA repair defect-cancer cells involves a number of DNA metabolic processes, including DNA replication, cell cycle checkpoints, and other DNA repair pathways. DNA repair defects can be found in hereditary as well as sporadic cancer. The extent of HR-defects in cancer cells needs to be determined by 
new functional testing of human cancers. As a consequence, the new cancer-specific synthetic lethal strategies can be tested on a larger pool of patients than just BRCA-mutation carriers. More efficient and specific approaches may be discovered, which would provide new avenues for the treatment DNA repair-defective human cancers.

\section{Acknowledgements}

We thank US Public Health Service Grants (Grant No. CA107640, to SNP), "Independent Innovation Foundation of Shandong University IIFSDU" (Grant No. 2010 TB017, to ZF), and the National Natural Science Foundation of China (Grant No.81172527, to ZF and SNP) for financial support.

\section{References}

1 Kaelin WG Jr. The concept of synthetic lethality in the context of anticancer therapy. Nat Rev Cancer 2005; 5: 689-698.

2 Nijman SM. Synthetic lethality: general principles, utility and detection using genetic screens in human cells. FEBS Lett 2011; 585: 1-6.

3 Farmer H, McCabe N, Lord CJ, et al. Targeting the DNA repair defect in BRCA mutant cells as a therapeutic strategy. Nature 2005; 434: 917-921.

4 Bryant HE, Schultz N, Thomas HD, et al. Specific killing of BRCA2-deficient tumours with inhibitors of poly (ADPribose) polymerase. Nature 2005; 434: 913-917.

5 Helleday T. Homologous recombination in cancer development, treatment and development of drug resistance. Carcinogenesis 2010; 31: 955-960.

6 Evers B, Helleday T, Jonkers J. Targeting homologous recombination repair defects in cancer. Trends Pharmacol Sci 2010; 31: 372-380.

7 Amir E, Seruga B, Serrano R, Ocana A. Targeting DNA repair in breast cancer: a clinical and translational update. Cancer Treat Rev 2010; 36: 557-565.

8 Satoh MS, Lindahl T. Role of poly (ADP-ribose) formation in DNA repair. Nature 1992; 356: 356-358.

9 Strom CE, Johansson F, Uhlen M, et al. Poly (ADP-ribose) polymerase (PARP) is not involved in base excision repair but PARP inhibition traps a single-strand intermediate. Nucleic Acids Res 2011; 39: 3166-3175.

10 Paques F, Haber JE. Multiple pathways of recombination induced by double-strand breaks in Saccharomyces cerevisiae. Microbiol Mol Biol Rev 1999; 63: 349-404.

11 Liu J, Heyer WD. Who's who in human recombination: BRCA2 and RAD52. Proc Natl Acad Sci U S A 2011; 108:
441-442.

12 Fujimori A, Tachiiri S, Sonoda E, et al. Rad52 partially substitutes for the Rad51 paralog XRCC3 in maintaining chromosomal integrity in vertebrate cells. EMBO J 2001; 20: $5513-5520$.

13 Feng Z, Scott SP, Bussen W, et al. Rad52 inactivation is synthetically lethal with BRCA2 deficiency. Proc Natl Acad Sci U S A 2011; 108: 686-691.

14 James CR, Quinn JE, Mullan PB, Johnston PG, Harkin DP. BRCA1, a potential predictive biomarker in the treatment of breast cancer. Oncologist 2007; 12: 142-150.

15 Turner N, Tutt A, Ashworth A. Hallmarks of 'BRCAness' in sporadic cancers. Nat Rev Cancer 2004; 4: 814-819.

16 Tomlinson GE, Chen TT, Stastny VA, et al. Characterization of a breast cancer cell line derived from a germ-line BRCA1 mutation carrier. Cancer Res 1998; 58: 3237-3242.

17 Bucher N, Britten CD. G2 checkpoint abrogation and checkpoint kinase-1 targeting in the treatment of cancer. $\mathrm{Br}$ J Cancer 2008; 98: 523-528.

18 Koniaras K, Cuddihy AR, Christopoulos H, Hogg A, O'Connell MJ. Inhibition of Chk1-dependent G2 DNA damage checkpoint radiosensitizes p53 mutant human cells. Oncogene 2001; 20: 7453-7463.

19 Chen CC, Kennedy RD, Sidi S, Look AT, D'Andrea A. CHK1 inhibition as a strategy for targeting Fanconi anemia (FA) DNA repair pathway deficient tumors. Mol Cancer 2009; 8: 24 .

20 Leijen S, Beijnen JH, Schellens JH. Abrogation of the G2 checkpoint by inhibition of Wee-1 kinase results in sensitization of p53-deficient tumor cells to DNA-damaging agents. Curr Clin Pharmacol 2010; 5: 186-191.

21 Hickson I, Zhao Y, Richardson CJ, et al. Identification and characterization of a novel and specific inhibitor of the ataxia-telangiectasia mutated kinase ATM. Cancer Res 2004; 64: 9152-9159.

22 Alabert C, Bianco JN, Pasero P. Differential regulation of homologous recombination at DNA breaks and replication forks by the Mrc1 branch of the S-phase checkpoint. EMBO J 2009; 28: 1131-1141.

23 Sorensen CS, Hansen LT, Dziegielewski J, et al. The cell-cycle checkpoint kinase Chk1 is required for mammalian homologous recombination repair. Nat Cell Biol 2005; 7: 195-201.

24 Powell SN, DeFrank JS, Connell P, et al. Differential sensitivity of p53(-) and p53(+) cells to caffeine-induced radiosensitization and override of G2 delay. Cancer Res 1995; 55: 1643-1648.

25 Shi W, Feng Z, Zhang J, et al. The role of RPA2 phosphorylation in homologous recombination in response to replication arrest. Carcinogenesis 2010; 31: 994-1002. 